\title{
BARRIERS TO HUMAN CONNECTIVITY AND THE DESIGN OF MORE COLLABORATIVE COWORKING SPACES
}

\author{
P. Mandeno ${ }^{\bowtie}$ and W. L. Baxter \\ Imperial College London, United Kingdom \\ $\bowtie$ p.mandeno17@imperial.ac.uk
}

\begin{abstract}
Coworking spaces - the most prevalent form of collaborative workplaces - are said to offer the ideal solution for a new generation of creative knowledge workers, balancing flexibility and independence with structure and community. Recent studies, however, highlight deficiencies as they relate to the promise of 'community' made by most coworking spaces. This work reports 16 barriers that impede the process of human connectivity in coworking spaces that emerged from indepth interviews with 26 coworkers. Suggestions are made for how these barriers might inform more effective workplace design.
\end{abstract}

Keywords: experience design, interaction design, human behaviour, human connectivity, coworking

\section{Introduction}

Since the turn of the century, a combination of organisational, social, technological and economic factors have radically transformed the way many people work. One of the most noticeable transformations is the rise of collaborative workplaces, particularly of the most common form, the coworking space (Kojo and Nenonen, 2017). Coworking spaces are seen to offer the ideal workplace solution for a new generation of creative knowledge workers seeking to balance flexibility and independence with structure and community (Fost, 2008; Uda, 2013). However, recent studies highlight deficiencies in community creation, with many coworkers - the users of these spaces - report feeling socially isolated (Spinuzzi et al., 2018). This suggests that traditional office concepts do not provide settings conducive to this new mode of work. Therefore, the design of office spaces for new collaborative modes of work, such as coworking, requires fundamentally rethinking interactions within the space as well as the factors that enhance or inhibit such interactions.

Explaining this phenomenon is the focus of this paper. Specifically, this study sought to identify, articulate and categorise the barriers to human connectivity within coworking spaces, where human connectivity is considered as both a process (the act of connecting) and an outcome (being connected) (Mandeno and Baxter, 2019). With a better understanding of the barriers to human connectivity in coworking spaces it should be possible to (re)design coworking spaces to account for these barriers and minimise their impact. The main contribution of this paper is the identification and articulation of 16 barriers that impede the process of human connectivity in coworking spaces. Suggestions are made regarding how acknowledging these barriers might inform the design of coworking spaces where human connectivity occurs more readily and ultimately collaboration increases.

Following an overview of relevant literature on coworking and human connectivity in the workplace the methodology of the study is presented. The paper concludes with the presentation and discussion 
of results along with suggestions regarding where design efforts may be focused in order to improve human connectivity outcomes.

\subsection{Coworking - background and coworker motivations}

The concept of sharing a workplace with others - generally for economic or social reasons - is not new. Studies that chart the evolution of coworking in its current form typically begin in the era of 'teleworking' in the 1960s and progress in a series of steps to the coworking solutions offered today (e.g. Kojo and Nenonen, 2017; Leclercq-Vandelannoitte and Isaac, 2016). Coining of the term 'coworking' as it is used today is widely attributed to entrepreneur Brad Neuberg who, in 2005, rented an office space that was too large for his personal needs and offered individual desks to others (Fost, 2008). This concept of "working alongside one another in flexible, shared work settings where desks can be rented on a daily, weekly or monthly basis" (Merkel, 2015, p. 122) took off. There are currently hundreds of thousands of coworkers working in thousands of coworking spaces in hundreds of cities worldwide (Spinuzzi et al., 2018).

Coworking's popularity seems to be the result of a combination of facilitating and motivating factors. Facilitating factors are those that make the current solution a viable alternative. For example, while early telecommunications technologies made it possible for people to work in a location other than their usual place of business, advances in digital technologies in the past two decades have made a much larger segment of workers completely mobile. Smartphone penetration is nearing $100 \%$ in many parts of the world meaning that a person is always reachable. As desktop computers are replaced by equally powerful laptops or tablets and cloud storage solutions become increasingly affordable and fast, it has become possible to work from anywhere that has an internet signal.

Motivating factors are specific to the individual and influence the choice of one workplace over another. A number of studies have explored the factors that motivate coworkers to pay to become members of coworking spaces (e.g. Brown, 2017; Kojo and Nenonen, 2017; Spinuzzi, 2012). While the specific motives identified may vary between studies, one motive identified consistently is the coworkers' desire to become part of a community of likeminded others. As many people who join coworking spaces are independent professionals, they do not have ready access to a community of colleagues as they might if they were part of a typical organisation. Although some studies have rightly challenged this notion of community as ill defined (e.g. Spinuzzi et al., 2018), it is clear from a review of coworking space websites that offering 'a sense of community' is a critical part of value proposition of most, if not all, coworking spaces. From the 1990s, some promise was seen in so-called 'third places' (Oldenburg, 1999) such as coffeeshops and libraries that offered a place to sit, internet access and a community of like minds. However, these did not provide the 'professionalisation' sought by many workers (Brown, 2017). Coworking spaces seek to fill this gap by offering a professional 'third place' but often fall short of fulfilling their socio-emotional promises to coworkers, particularly as they relate to offering a sense of community.

\subsection{Human connectivity and workplace design}

Feeling connected to others is generally understood to be a fundamental human need (Baumeister and Leary, 1995). The social and physiological benefits of being better connected to others, as well as the detrimental effects of being poorly connected, are vast and well documented (e.g. Holt-Lunstad et al., 2015). Early studies that explored the effects of good or poor human connectivity typically focused on the personal lives of individuals. In more recent decades, researchers have increasingly considered organisational settings and their results point to equally alarming levels of social isolation in the workplace (Murthy, 2017).

Beyond purely socio-emotional outcomes of human connectivity, cognitive and functional outcomes (Mandeno and Baxter, 2019) make the case for being better-connected in the workplace all the more compelling. For example, better-connected people have more successful careers (Useem and Karabel, 1986), are more effective at completing tasks (Kadushin, 2004), have better access to valuable information (Inkpen and Tsang, 2005), are included in better opportunities, and are better able to exert influence over others (Burt, 2000). Better-connected people are not only happier and healthier at work, they are also more effective and successful. 
Although the benefits of human connectivity are well understood, developing a workplace where strong human connectivity occurs is a continuing issue for coworking spaces (Spinuzzi, 2012). In part, this is because those in the space come from many different domains, backgrounds and life-stages and so lack touchpoints, norms and shared identities common within traditional workplaces. Because of this, it is not enough to simply collocate people under one roof and expect them to connect. The way people interact with the space, and ultimately each other, has to be rethought while simultaneously taking account of the diversity of their needs and expectations. The complexity of this task also highlights the opportunity within design. The redesign of a space for collaboration, such as a coworking space, means that services will need to be implemented, products made for multiple uses, infrastructure that enables connection while maintaining privacy introduced, and experiences created that provide common touchpoints between workers and many more. In this work, we adopt a humancentric view of the barriers to human connectivity within the coworking context as a first step towards designing spaces where people develop more quality connections. In doing so, people will reap the benefits of improved human connectivity and increase their potential for collaboration.

\section{Methodology}

This study sought to understand the subjective experience of coworkers and, more specifically, identify the barriers that impede the process of human connectivity in coworking settings. A qualitative inductive approach was employed with data collected by way of in-depth semi-structured interviews.

The interview script began with open questions about the general experience of coworking and progressed to include more specific questions relating to the experience of human connectivity. Participants were asked to think specifically about the barriers they had experienced in each of the 5 phases of human connectivity (Mandeno and Baxter, forthcoming).

In order to achieve a broad perspective from individuals who cowork, the selected sample was intentionally diverse (Creswell, 2007). In total, 26 participants (18 female) were recruited for the study. Their ages ranged from 27 to 52 years and they were located in 14 cities (10 countries). 17 participants had hotdesk memberships, four had permanent desks and five had private offices. The duration of their memberships ranged from three months to 15.5 years and their company sizes ranged from sole proprietors (e.g. lone freelancers) to several thousand person multinational organisations. Participants were recruited through a LinkedIn advertisement and subsequent snowball sampling. All were required to be 18 years or older and have worked at a coworking space for 3 months or more.

Interviews were conducted face-to-face when possible and otherwise via Skype with data collection taking place between August 2018 and January 2019. The average interview duration was 43 minutes. Interviews were recorded and transcribed verbatim and subsequently coded.

Data were analysed inductively following the 6-phases of reflexive thematic analysis (Braun et al., 2019). Initially, interview transcripts were read in order to familiarize the authors with their contents and make initial notes. Next, codes were generated that began to organise the data into chunks. Transcribed interviews were coded using NVivo 12 for Mac software. Codes were then combined into themes that grouped the codes. Themes were critically analysed relative to the data and the research question in a process of further revision and definition. Once satisfied with the resulting themes, definitions were constructed for each theme together with contextually-relevant explanations, examples and considerations.

\section{Results and discussion}

Most coworking spaces make the promise of becoming part of a thriving community of likeminded individuals yet the experience of coworkers seems to be quite different, as stated rather bluntly by one participant (P25) of this study. When asked to comment on the claim made on the website of the coworking space of which he was a member - 'make new connections and build your career as part of a thriving community of mobile, flexible professions'- he replied, "Yeah, it's bullshit, yeah". This was the overwhelming sentiment of participants interviewed for this study. It seems that although coworking spaces believe they are creating and offering access to thriving communities, the reality of the coworker experience is quite the opposite. These results reveal many factors that are to blame. 
Analysis of the data resulted in 16 distinct barriers (Figure 1) organised into two broad categories (situational and individual), and subsequently divided into sub-categories (group, setting, contact and connector).

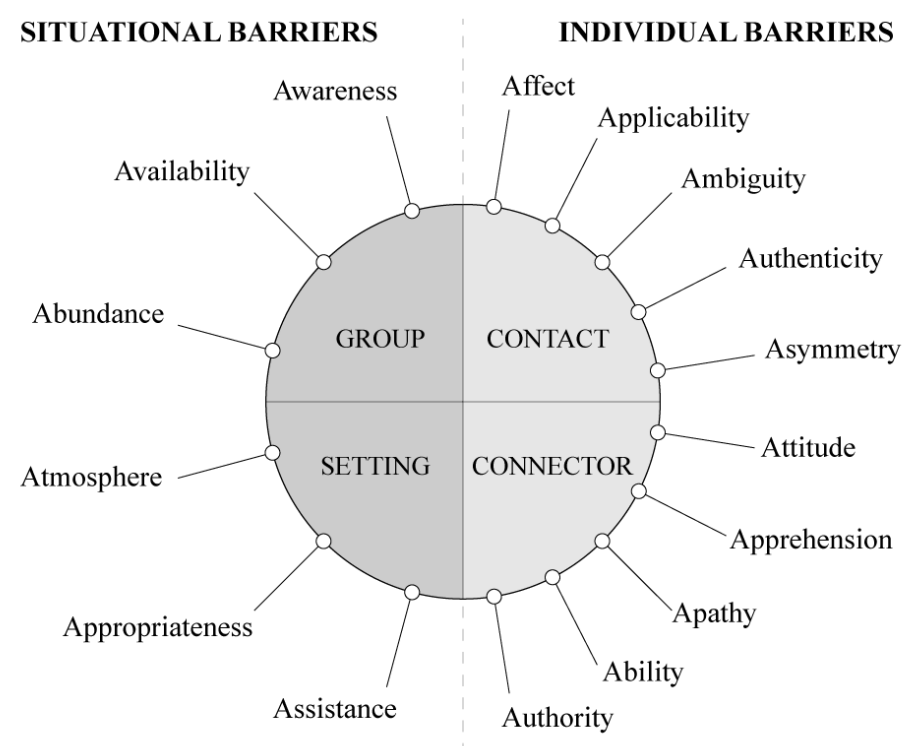

Figure 1. Barriers to human connectivity in coworking spaces including categories and sub-
categories

Situational barriers include the overall group of individuals (all members of a coworking space) within which a person is seeking to connect and the setting in which the connecting is intended to take place. Individual barriers include those that are a function of the dyad, namely the contact (the target of connectivity efforts) and the connector (the person seeking to connect). Here, each barrier is explained in more detail. Suggestions are provided for a human-centric design approach to addressing each barrier.

Three group-related barriers were identified, referring to the group to which the contact and connector belong (all members of their coworking space) - namely: awareness, availability and abundance.

1. Awareness refers to the connector being unaware of which contacts are utilising the coworking space and what value each contact might represent. This was described by one participant (P06): "But it's not clear from the outside who's doing what and who might be the one you are looking for." Lack of awareness appeared to be a function of two key factors. Firstly, because of the member-diversity common to most coworking spaces, it was difficult for members to predict who else they are sharing the space with. Unlike a traditional office space where everyone belongs to the same company or industry, many coworking spaces attract members from a variety of different industries, and it is not clear who does what. Secondly, as the general trend of increased job mobility continues (Culpin et al., 2015), it is likely that a contact's current role does not represent their entire career. In their 2017 global survey, Deskmag noted the average age of coworkers was over 36 years (Foertsch, 2017), close to the average of 40 years in the current study. Participants commented they were had considerable experience that was unrelated to their current venture (e.g. P10: corporate strategist currently in personal development). In summary, these two factors make it difficult to for connectors to know all of the relevant information about the contacts they share the space with. Addressing this barrier will require the design of services that provide more complete coworker profiles. These may be passive, requiring effort on the part of connectors to search for example, or active in which they play a more effective match-making role.

2. Availability refers to the contact being physically unavailable to the connector, or vice versa, and comes down to "they not being there or me not being there" (P15). Lack of availability arose not only from who was using the space but how they use it. A key factor that amplified this barrier was that most coworkers are not beholden to company norms such as workday 
start and end times or the number and timing of breaks they take throughout the day. Because of this, the likelihood of the connector and the contact being in the same place at the same time diminishes, subsequently reducing their ability to connect. Addressing this barrier requires devising ways to align the schedules of coworkers within a coworking space or create touchpoints (e.g. activities) through which the probability of seeing someone increases.

3. Abundance refers to the connector being overwhelmed by the number of contacts with whom they may potentially connect or wish to stay connected. This barrier was most apparent in large coworking spaces. The average membership base of the coworking spaces occupied by participants of this study was 383 members. While such a large potential pool of contacts may provide many potential connections, several participants mentioned that they were simply overwhelmed. They were either confused regarding where to start or guilty that they knew too many people but did not have time to keep in touch with them all. This is not surprising when the extant literature suggests limits to the number of connections a person can effectively manage (Dunbar, 1998). Another explanation is provided by Schwartz (2004) who refers the 'paradox of choice' where too much choice can have negative consequences, even leading to anxiety and depression. As most coworking spaces are commercial enterprises and voluntary reduction of member numbers is unlikely, addressing this barrier is challenging. Relevant solutions may help coworkers to focus on more manageable subsets of the entire population within a coworking space. Additionally, services or activities that reduce a coworker's cognitive load (remaining connected to existing contacts) would likely prove beneficial.

Three setting-related barriers were identified. These barriers related to the overall coworking setting in which human connectivity was taking place - namely: atmosphere, appropriateness and assistance.

4. Atmosphere is an all-encompassing barrier that refers to aspects of the physical space that may hinder the process of connecting. Participants mentioned everything from seating to lighting (P20) to the coffee space (P03) to spaces feeling cheap (P07), claustrophobic (P11) and lacking privacy (P12). Such barriers are well documented in the literature in studies that highlight how human connectivity may be impeded by the lack of: proximity (Allen, 2007); privacy (Bernstein and Turban, 2018); comfort (Fayard and Weeks, 2007); and even by the physical design of objects in the space (Blumenthal, 2007). There is clearly great scope for addressing the range of issues highlighted by this barrier. As well as the basic requirements of comfort and safety, balance must be struck between creating spaces in which coworkers naturally bump into each other and that also afford privacy and intimacy. Because of the diversity of members occupying most coworking spaces, designers should be cautious of applying the same rules that guide the design of traditional office spaces.

5. Appropriateness refers to the connector being unsure about what behaviours are appropriate when it comes to engaging with contacts. This is about "what is alright to do, what's permissible behaviour" (P15). Coworking member bases are typically diverse in terms of members' cultural background as well as their industry or domain. For example, of the participants interviewed in this study, almost half (44\%) were not working in their country of origin. Additionally, coworking spaces are increasingly attracting corporate members who have very different priorities from the traditional coworking population of independent knowledge workers. Acceptable behaviour in one culture or sector is often unacceptable in another. This can lead to anxiety ('what is the right approach?') and may prevent a connector from seeking to connect. Although some coworking spaces offer social events to help their members to find common ground, these are not always appealing to all members and can also result in segmentation. Coworking space owners or hosts often default to what they personally prefer and expect their members to think or want the same when in fact the coworkers they host can be quite different to themselves (Spinuzzi, 2012). Design solutions to address appropriateness must combine a better understanding of the expectations of all members with clearer communication regarding what is acceptable behaviour.

6. Assistance refers to the people running a coworking space and the role they fail to play in helping members of their coworking space to connect. This is often about the perceived priorities of coworking space owners or host where "they're focusing too much on being a 
real estate company versus a community-creator" (P07). The majority of coworking spaces mention joining their thriving community (of coworkers) as a key part of their value proposition. However, most participants of the current study believed that this was mostly sales-speak and the reality was much different. Coworking spaces seemed very attentive to prospective members but as soon as they had signed their membership contracts, spaces were only interested in their recurring monthly fee. In spaces where assistance was given, it often came down to a lone individual (e.g. front-desk host) taking it upon themselves to connect members. Addressing this barrier requires that coworking space owners make 'connecting others' a more integral and purposeful part of their culture beyond the moment of a new coworker signing up. Hosts who are present day-to-day should receive the appropriate resources (e.g. time, training) and tools to fulfil this role most effectively.

Five contact-related barriers were identified. These barriers relate to the target of connection namely: affect, applicability, ambiguity, authenticity and asymmetry.

7. Affect refers to a connector disliking a potential contact, even if they could be valuable. Several participants were very explicit about this being a critical deciding factor, exemplified by P07 who said "basically I try to never work with assholes to any degree". Affect and competence are recognised in the literature to offer the best indication of the extent to which a person will choose to connect with another (Fiske et al., 2007). In any encounter, the connector is asking 'are you a good person?' (affect) and 'can you do what you say?' (competence). Casciaro \& Lobo (2008) suggest that likeability will always trump competence. That is, regardless of how competent a contact is, if the connector does not like them, the likelihood of a successful connection is very low. An important consideration for coworking spaces is the cultural diversity of their members. Culturally diverse people may bring different social norms and behaviours (McCrae and Terracciano, 2005) so common measures of likeability are not always as reliable. Addressing the barrier of affect directly will be challenging (you can't make one person like another). Solutions may instead focus on creating opportunities for coworkers to learn more about each other, and appreciate their differences as positive, not negative.

8. Applicability refers to a connector believing a contact is not relevant to them. This barrier generally resulted in some form of judgement that deemed the other person to be irrelevant. Participants reported lack of applicability in several forms, including: moral applicability e.g."I'm talking more about like profound solutions to the problems in the world and they were making an app so you can buy more hats" (P07); transactional applicability - e.g. "the connections I need tend to be in big cities, not here" (P13); and applicability of character e.g. "If their [LinkedIn] profile is kind of incomplete or dodgy then you know that this person has some other issues" (P18). In situations where a connector considers the contact to be an outgroup member, the connector will be strongly biased against the contact (Castano et al., 2002) and is less likely to trust them (Wu et al., 2011). Design solutions to overcome this barrier should focus on services and activities that help coworkers to discover what they have in common to overcome immediate judgements based on their differences.

9. Ambiguity refers to a connector being unsure how to leverage the apparent value inherent in a connection. This is articulated by P16 as: "They might be useful or they might not, and sometimes it's really hard to know". This sentiment was most common for creative knowledge workers in the sample who found potential in many others. This creates an interesting paradox. The more open a person is to all types of others, the more likely they are to amass ambiguous connections where the value inherent in the connection is unclear. Research suggests that there are limits to the number of connections a person can maintain (Dunbar, 2011) so such people run the risk of wasting cognitive and emotional energy on potentially irrelevant connections. Addressing this barrier will require helping people to more quickly and effectively identify the value inherent in a connection and manage increasingly large collections of potentially useful contacts.

10. Authenticity refers to the connector believing the contact is not genuine or has ulterior motives. Lack of authenticity manifest in a variety of ways as reported by participants of this study with inauthentic people being described as "creepy and manipulative" (P20), "transactional" (P22) and "not genuine" (P17) for example. Authenticity helps to build trust which is a critical 
component of quality human connections (Stephens et al., 2012). Again, because of the inherent diversity within the membership of coworking spaces, diversity of values and behaviours can also be expected. While some contacts may indeed be inauthentic, connectors run the risk of ruling someone out simply because their behaviour is unfamiliar. In addressing this barrier, attention might be placed on helping coworkers to be curious about their cultural differences and be open to connections that may be valuable in ways other than first thought.

11. Asymmetry refers to a perceived imbalance in the value exchange in the dyad with the connector believing they were "giving a lot while the other side was just kind of taking and not giving back" (P23). Asymmetry was most acute for coworkers who were known to possess a specific skill that many people valued (e.g. legal or tax experience), or those who were further in their entrepreneurial journey and could therefore speak from experience. Those with skills and experience were often the target of a disproportionate number of requests from other coworkers who wanted free advice. Additionally, several participants reported that asking for help is easier for some people than it is for others and that saying 'no' is often difficult. Research indeed shows that some people are more comfortable saying 'no' than others (Flynn and Lake, 2008). Together, these factors exacerbated asymmetry, if a person was the target of many requests and could not say no. This barrier could be addressed by enabling proportional value, finding new forms of value to exchange or introducing policies to protect those in high demand.

Finally, five connector-related barriers were identified - namely: attitude, apprehension, apathy, ability and authority.

12. Attitude refers to the connector's mindset getting in the way of a successful connection. Attitude was affected by a connector's personality, often related to their culture - e.g. "the prevailing mentality in our culture is just kind of solitary" (P20), or by a person's general mindset toward others - e.g. "I don't think I've ever been that curious about the people here" (P19). This is reflected in the literature that suggests openness may be influenced by a person's culture (McCrae and Terracciano, 2005) and is linked to their sense of curiosity (Park et al., 2004). Because of the diversity of coworkers in most coworking spaces, cultural and personality differences should be expected. Although changing personalities and accommodating all cultural differences may be difficult, this barrier may be addressed by initiatives that foster more open and curious coworking cultures, requiring coworking hosts to lead by example.

13. Apprehension refers to the connector feeling anxious or generally having second thoughts about connecting to others. The cause of participants' apprehension included: wasted effort e.g. "I probably won't see him tomorrow so what's the point?" (P25); confidence and fear of rejection - e.g. "It's about self-esteem and caring what other people think" (P15); and fear of burdening someone - e.g. "It might be too much to ask" (P26). Humans are social beings, strongly motivated to connect to one another (Baumeister and Leary, 1995). A connector's fear that a prospective contact will reject them can therefore be the source of great anxiety (Downey and Feldman, 1996). Interestingly, when the tables were turned, the same participants took the opposite view. For example, someone afraid of approaching others would be very open to others approaching them. Addressing this barrier may be achieved through initiatives that bolster the confidence of coworkers. Positive experiences may appease fears and make coworkers less apprehensive. Similarly, interventions that explicitly or implicitly grant permission to engage with others are likely to have a positive impact in addressing this barrier.

14. Apathy refers to a connector not being proactive enough, or "If you don't go out and make an effort you lose out on connections" (P14). Several participants mentioned being aware they could (or should) do more than what they were currently doing, A common insight was that people made more of an effort when first joining a coworking space - e.g. "we used to speak to someone new every week" (P19) - but their interest and effort waned over time. Two reasons provided by this same participant were "you forget when you are caught up in your own work", and "you also want your privacy, so sometimes you don't want to have to talk to everyone". The responses of participants in the current study suggest that this barrier may be explained in terms of capacity (when push comes to shove, work takes priority) and motivation (the benefit is unclear, so this is not urgent). As studies from other settings have 
shown, people can underestimate the benefit derived even from seemingly insignificant social exchanges (Epley and Schroeder, 2014; Sandstrom and Dunn, 2014) and are less likely to make an effort. Effective design solutions to address this barrier might help coworkers to develop ritualised behaviours that help to integrate 'connecting to others' into their everyday routines rather than seeing it as an additional task.

15. Ability refers to the connector lacking the skills required to connect effectively to others. One participant (P15) referred to connecting to others as a "practiced skill", which, when lacking, led to a connector "not knowing what to say". Another (P20) likened connecting to the "selling of ideas" which was "not always very easy to do". What is clear from the present study, and supported by the literature (e.g. Baker, 2000), is that some people indeed seem more skilled than others in social situations. While lack of ability may be real, prior studies suggest that in some cases it is merely a person's perception (Jones, 1986). Perceived or actual lack of ability may be exacerbated if we consider the cultural diversity already discussed as some cultures tend to fare better in social encounters (McCrae and Terracciano, 2005). Lack of ability may be addressed by interventions that improve the overall connectivity skillset of coworkers and by creating risk-free space for them to practice.

16. Authority refers to the connector not being taken seriously by the contact. This was described by one participant as "not wielding the power" (P20) necessary to make collaboration happen. The age range of participants in the current study was 27 to 52 years which suggests significant diversity of seniority. Coworking spaces are increasingly attracting older coworkers (Foertsch, 2017), some of whom may bring with them a traditional hierarchical mindset, leading them to discredit younger counterparts. As well as age (and related experience), gender and cultural differences may also lead to such discrimination in such settings (e.g. Burt, 2000). Interestingly, research suggests the reverse may also be true, where older coworkers are not taken seriously or perceived as competent by younger colleagues (Guillén and Kunze, 2019). Efforts to address lack of authority in either direction will require finding ways to dismantle traditional power structures and create opportunities for coworkers at each end of the dimensions of authority to recognise their respective value to each other.

\section{Conclusion}

Coworking spaces are said to offer the ideal setting for a new generation of flexible workers, due in part to the thriving communities of diverse members who occupy them. However, as previous studies have suggested (e.g. Spinuzzi, 2012), coworking spaces are not delivering on their promise of community. This study corroborates these findings and goes further to identify 16 specific barriers, divided in to four distinct categories, that help to explain why coworkers are failing to effectively connect to each other. With this deeper and more specific level of understanding, designers of all facets of the coworking experience will be better able to create solutions that lead to improved human connectivity, which should, in turn, increase the likelihood of collaboration.

Three nuances of these findings should be noted. Firstly, while there generally tends to be one dominant barrier that can best explain a connector's failure to connect in a given context, the 16 barriers should not be considered to be mutually exclusive. Multiple barriers can be working in unison, amplifying their relative effect. Secondly, barriers may have more effect in one phase of the human connectivity process than in others (Mandeno and Baxter, 2019). Thirdly, it is critical to note that barriers arise from several dimensions of the coworking experience including the setting, the overall group to which a coworker belongs, and factors relating to the individuals seeking to connect. The selection of participants and workplaces for this study was deliberately diverse. This aided in the compilation of a comprehensive set of barriers yet made it difficult to find correlation on the basis of a participant's culture. Cultural difference may influence a barrier's relative effect. Future research might explore this in more detail.

The designers of many coworking spaces seem to rely on tacit knowledge gained in traditional offices. This study shows that coworking settings are unique and greater effort is required to enable social norms and collegiate work to take place, supported by more successful interactions (Buchanan, 2010). Writing about workplace settings, Wineman and Barnes (2018) suggest that "the design question becomes how to provide the most effective collaborative opportunities given the unique characteristics 
of an organization" (p.188). What this study shows is that, in the case of designing coworking spaces, attention should instead be focused on the unique characteristics of the individuals that occupy them.

\section{References}

Allen, T.J. (2007), "Architecture and Communication among Product Development Engineers", California Management Review, Vol. 49 No. 2, pp. 23-41. https://doi.org/10.2307/41166381, SAGE Publications Inc.

Baker, W. (2000), Networking Smart: How to Build Relationships for Personal and Organizational Success, iUniverse, Incorporated.

Baumeister, R.F. and Leary, M.R. (1995), "The need to belong: Desire for interpersonal attachments as a fundamental human motivation", Psychological Bulletin, Vol. 117 No. 3, pp. 497-529. https://doi.org/ 10.1037/0033-2909.117.3.497

Bernstein, E.S. and Turban, S. (2018), "The impact of the 'open' workspace on human collaboration", Philosophical Transactions of the Royal Society of London. Series B, Biological Sciences, Vol. 373 No. 1753. https://doi.org/10.1098/rstb.2017.0239

Blumenthal, K. (2007), Grande Expectations: A Year in the Life of Starbucks' Stock, Crown Business Publications, New York.

Braun, V. et al. (2019), "Thematic Analysis", In: Liamputtong, P. (Ed.), Handbook of Research Methods in Health Social Sciences, Springer Singapore, Singapore, pp. 843-860. https://doi.org/10.1007/978-981-10-5251-4_103

Brown, J. (2017), "Curating the 'Third Place'? Coworking and the mediation of creativity", Geoforum, Vol. 82, pp. 112-126. https://doi.org/10.1016/j.geoforum.2017.04.006

Buchanan, R. (2010), "Design Research and the New Learning", Design Issues, Vol. 17 No. 4, pp. 3-23. https://doi.org/10.1162/07479360152681056

Burt, R.S. (2000), "The Network Structure Of Social Capital”, Research in Organizational Behavior, Vol. 22, pp. 345-423. https://doi.org/10.1016/s0191-3085(00)22009-1

Casciaro, T. and Lobo, M.S. (2008), "When competence is irrelevant: The role of interpersonal affect in taskrelated ties", Administrative Science Quarterly, Vol. 53 No. 4, pp. 655-684. https://doi.org/10.2189/ asqu.53.4.655, SAGE Publications Sage CA: Los Angeles, CA.

Castano, E. et al. (2002), "Who May Enter? The Impact of In-Group Identification on In-Group/Out-Group Categorization", Journal of Experimental Social Psychology, Vol. 38 No. 3, pp. 315-322. https://doi.org/ 10.1006/jesp.2001.1512, Elsevier.

Creswell, J.W. (2007), Qualitative Inquiry and Research Design: Choosing among Five Approaches, Second, Sage publications.

Culpin, V. et al. (2015), "How have careers changed? An investigation of changing career patterns across four generations", Journal of Managerial Psychology. https://doi.org/10.1108/jmp-07-2014-0210, Emerald Group Publishing Limited.

Downey, G. and Feldman, S.I. (1996), "Implications of Rejection Sensitivity for Intimate Relationships", Journal of Personality and Social Psychology, Vol. 70 No. 6, pp. 1327-1343. https://doi.org/10.1037/00223514.70.6.1327

Dunbar, R.I.M. (1998), “The social brain hypothesis", Evolutionary Anthropology: Issues, News, and Reviews, Vol. 6 No. 5, pp. 178-190. https://doi.org/10.1002/(sici)1520-6505(1998)6:5, Wiley-Blackwell.

Dunbar, R.I.M. (2011), How Many Friends Does One Person Need? Dunbar's Number and Other Evolutionary Quirks, Harvard University Press, United States.

Epley, N. and Schroeder, J. (2014), "Mistakenly seeking solitude", Journal of Experimental Psychology: General, American Psychological Association, Vol. 143 No. 5, p. 1980. https://doi.org/10.1037/a0037323

Fayard, A.-L. and Weeks, J. (2007), "Photocopiers and Water-coolers: The Affordances of Informal Interaction", Organization Studies, SAGE Publications Ltd, Vol. 28 No. 5, pp. 605-634. https://doi.org/10.1177/ 0170840606068310

Fiske, S.T., Cuddy, A.J.C. and Glick, P. (2007), "Universal dimensions of social cognition: Warmth and competence", Trends in Cognitive Sciences, Elsevier, Vol. 11 No. 2, pp. 77-83. https://doi.org/10.1016/j. tics.2006.11.005

Flynn, F.J. and Lake, V.K.B. (2008), "If you need help, just ask: underestimating compliance with direct requests for help", Journal of Personality and Social Psychology, Vol. 95 No. 1, p. 128. https://doi.org/10.1037/00223514.95.1.128, American Psychological Association.

Foertsch, C. (2017), “The Members: Who Works in Coworking Spaces?", Deskmag, available at: http://www.deskmag.com/en/members-of-coworking-spaces-demographics-statistics-global-survey-cowor kers-research-2017.

Fost, D. (2008), "They're Working on Their Own, Just Side by Side", New York Times, available at: https://www.nytimes.com/2008/02/20/business/businessspecial2/20cowork.html. 
Guillén, L. and Kunze, F. (2019), "When age does not harm innovative behavior and perceptions of competence: Testing interdepartmental collaboration as a social buffer", Human Resource Management, John Wiley \& Sons, Ltd, Vol. 58 No. 3, pp. 301-316. https://doi.org/10.1002/hrm.21953

Holt-Lunstad, J. et al. (2015), "Loneliness and Social Isolation as Risk Factors for Mortality: A Meta-Analytic Review", Perspectives on Psychological Science, Vol. 10 No. 2, pp. 227-237. https://doi.org/10.1177/ 1745691614568352, SAGE Publications Inc.

Inkpen, A.C. and Tsang, E. (2005), "Social Capital, Networks, and Knowledge Transfer", Academy of Management Journal, Vol. 30 No. 1, pp. 146-165. https://doi.org/10.2307/20159100

Jones, G.R. (1986), "Socialization Tactics, Self-Efficacy, and Newcomers' Adjustments to Organizations", The Academy of Management Journal, Vol. 29 No. 2, pp. 262-279. https://doi.org/10.2307/256188, Academy of Management.

Kadushin, C. (2004), “Too Much Investment in Social Capital?”, Social Networks, Vol. 26 No. 1, pp. 75-90, https://doi.org/10.1016/j.socnet.2004.01.009

Kojo, I. and Nenonen, S. (2017), "Evolution of co-working places: drivers and possibilities", Intelligent Buildings International, Vol. 9 No. 3, pp. 164-175. https://doi.org/10.1080/17508975.2014.987640, Taylor \& Francis.

Leclercq-Vandelannoitte, A. and Isaac, H. (2016), "The new office: how coworking changes the work concept", Journal of Business Strategy, Vol. 37, pp. 3-9. https://doi.org/10.1108/jbs-10-2015-0105

Mandeno, P. and Baxter, W.L. (2019), "Design for human connectivity: A framework and research agenda", International Association of Societies of Design Research Conference 2019 Design Revolutions.

McCrae, R.R. and Terracciano, A. (2005), "Personality profiles of cultures: aggregate personality traits", Journal of Personality and Social Psychology, Vol. 89 No. 3, pp. 407. https://doi.org/10.1037/0022-3514.89.3.407, American Psychological Association.

Merkel, J. (2015), "Coworking in the city", Ephemera, [United Kingdom]

Murthy, V. (2017), "Work and the Loneliness Epidemic", Hbr.Org, available at: https://hbr.org/coverstory/2017/09/work-and-the-loneliness-epidemic

Oldenburg, R. (1999), The Great Good Place: Cafes, Coffee Shops, Bookstores, Bars, Hair Salons, and Other Hangouts at the Heart of a Community, Da Capo Press.

Park, N., Peterson, C. and Seligman, M.E.P. (2004), "Strengths of character and well-being", Journal of Social and Clinical Psychology, Guilford Press, Vol. 23 No. 5, pp. 603-619. https://doi.org/10.1521/jscp.23.5.603.50748

Sandstrom, G.M. and Dunn, E.W. (2014), "Social Interactions and Well-Being: The Surprising Power of Weak Ties", Personality and Social Psychology Bulletin, SAGE Publications Inc, Vol. 40 No. 7, pp. 910-922. https://doi.org/10.1177/0146167214529799

Schwartz, B. (2004), The Paradox of Choice : Why More Is Less, HarperCollins, New York.

Spinuzzi, C. (2012), "Working Alone Together: Coworking as Emergent Collaborative Activity", Journal of Business and Technical Communication, Vol. 26 No. 4, pp. 399-441. https://doi.org/10.1177/10506519 12444070, SAGE Publications Inc.

Spinuzzi, C. et al. (2018), “'Coworking is about community' but what is 'community' in coworking?”, Journal of Business and Technical Communication. https://doi.org/10.1177/1050651918816357, SAGE Publications.

Stephens, J.P., Heaphy, E. and Dutton, J.E. (2012), "High quality connections", The Oxford Handbook of Positive Organizational Scholarship, Oxford University Press Oxford, pp. 385-399. https://doi.org/10.1093/oxfordhb/ 9780199734610.013.0029

Uda, T. (2013), What Is Coworking? A Theoretical Study on the Concept of Coworking, No. 2013-265. https://doi.org/10.2139/ssrn.2937194

Useem, M. and Karabel, J. (1986), "Pathways to Top Corporate Management", American Sociological Review, Vol. 51 No. 2, pp. 184-200. https://doi.org/10.2307/2095515, Sage Publications, Inc.

Wineman, J.D. and Barnes, J. (2018), "Workplace Settings”, In: Devlin, A.S. (Ed.), Environmental Psychology and Human Well-Being, Elsevier, pp. 167-192. https://doi.org/10.1016/b978-0-12-811481-0.00007-X

Wu, Y., Leliveld, M.C. and Zhou, X. (2011), "Social distance modulates recipient's fairness consideration in the dictator game: An ERP study", Biological Psychology, Elsevier, Vol. 88 No. 2-3, pp. 253-262. https://doi.org/10.1016/j.biopsycho.2011.08.009 